\title{
DINÂMICA DA DORMÊNCIA DE GEMAS DE MACIEIRA 'IMPERIAL GALA' DURANTE O OUTONO E INVERNO EM REGIÃO DE BAIXA OCORRÊNCIA DE FRIO ${ }^{1}$
}

\author{
RUY INACIO NEIVA DE CARVALHO² \& FLÁVIO ZANETTE ${ }^{3}$
}

\begin{abstract}
RESUMO - O objetivo deste trabalho foi determinar a dinâmica da dormência de gemas de um ano de macieira 'Imperial Gala' com ou sem frio suplementar durante o outono e o inverno, cultivadas em região de baixa ocorrência de frio. Os ramos foram coletados em Porto Amazonas-PR, em intervalos de 21 dias, de abril a agosto (19-04, 10-05, 31-05, 21-06, 12-07, 02-08 e 23-08) e receberam ou não tratamento com frio suplementar de 1.440 horas à temperatura de 4 a $7^{\circ} \mathrm{C}$. A avaliação da dormência foi realizada pelo teste biológico de estacas de nós isolados (temperatura de $25^{\circ} \mathrm{C}$ e fotoperíodo de 16 horas) por meio dos parâmetros: tempo médio para brotação (TMB), velocidade de brotação (VB), taxa final de brotação (TF), taxa de brotações vigorosas (TBV) e tempo médio para aparecimento de folhas abertas (TMFA). A dormência mais intensa das gemas de um ano ocorreu em 12 de julho. A aplicação de 1.440 horas de frio suplementar de 4 a $7^{\circ} \mathrm{C}$ foi efetivo para a redução do tempo médio de brotação das gemas. Autilização da variável TBV nos testes de estacas de nós isolados foi uma importante forma de avaliação da capacidade real de desenvolvimento da gema, diminuindo-se a interferência do corte da estaca como estimulador de início de desenvolvimento.
\end{abstract}

Termos para Indexação: Malus domestica Borkh., brotação, ecofisiologia, endodormência.

\section{DORMANCY DYNAMICS OF 'IMPERIAL GALA' APPLE TREE BUDS DURING AUTUMN AND WINTER IN A REGION OF LOW CHILL OCCURENCE}

\begin{abstract}
This work aimed to evaluate the dormancy dynamics of one year old apple tree buds with or without supplementary chill during autumn and winter, in a region of low chill occurence. The stems were collected in Porto Amazonas, Parana State, Brazil, at intervals of 21 days from April to August $\left(04 / 19,05 / 10,05 / 31,06 / 21,07 / 12,08 / 02\right.$ and $08 / 23$ ) and were treated or not with 1,440 hours of chill (4 to $7^{\circ} \mathrm{C}$ ). The evaluation of dormancy was observed by the biological test of single node cuttings under controlled conditions $\left(25^{\circ} \mathrm{C}\right.$ and long days of 16 hours $)$ by following parameters: average time for budburst (TMB), velocity of budburst (VB), final rate of budburst (TF), rate of vigorous budburst (TBV) and average time for appereance of opened leaves (TMFA). The more intense dormancy occured on July, 12 . The chill treatment with 1,440 hours ( 4 to $7^{\circ} \mathrm{C}$ ) was effective in reducing the average time for budburst. The use of TBV in single node cuttings test was an important method to evaluate the real capacity of bud development, reducing the interference of cut like an initial development estimulator.
\end{abstract}

Index Terms: Malus domestica Borkh., budburst, ecophysiology, endodormancy.

\section{INTRODUÇÃO}

O estudo da fisiologia da dormência de gemas é fundamental para o sucesso do manejo da superação da dormência, técnica necessária para a exploração racional de espécies de clima temperado no Brasil, em especial em regiões de baixa ocorrência de frio.

Durante as estações desfavoráveis, as plantas limitam ou cessam seu crescimento de forma a permitir a sobrevivência em períodos de escassez de água ou de baixas temperaturas. Apesar de a planta não apresentar crescimento visível, as atividades metabólicas essenciais continuam a ocorrer com intensidade reduzida (Petri et al., 1996).

Segundo Lang et al. (1987), a dormência é classificada em três fases: paradormência, endodormência e ecodormência. A endodormência ocorre nos meses mais frios, em que a paralisação do desenvolvimento da gema é resultante de uma série de eventos bioquímicos e fisiológicos que acontecem em tecidos meristemáticos ou regiões muito próximas; a paradormência antecede a endodormência e é resultante da influência de outro órgão do vegetal sobre a gema, e a ecodormência ocorre após a endodormência, quando a brotação da gema não ocorre devido a fatores extrínsecos à planta.

A baixa temperatura é um fator ambiental de grande influência na endodormência de gemas (Crabbé \& Barnola, 1996). As respostas das gemas de macieira em relação às baixas temperaturas são variadas de acordo com a idade das gemas (Zanette et al., 2000) e influenciam no crescimento e na sobrevivência de plantas em casa de vegetação (Pereira et al., 2001b). Os processos fisiológicos internos envolvidos na entrada e saída da endodormência podem estar relacionados com modificações na estrutura celular (Larcher, 2000), alterações do metabolismo energético na gema, como a atividade de enzimas e a síntese de nucleotídeos
(Bonhomme et al., 2000), conteúdo de proteínas (Tamura et al., 1998), fluxo de carboidratos, suprimento de nutrientes e translocação de reservas a curta distância (Erez, 2000; Marquat et al., 1999), regulação hormonal (Stafstrom, 2000), bem como com uma relação entre todos estes fatores (Crabbé \& Barnola, 1996), resultando numa dinâmica de resposta à ação do frio muito variada.

O objetivo deste trabalho foi determinar a dinâmica da dormência de gemas em ramos de um ano de macieira 'Imperial Gala' com ou sem frio suplementar, durante o outono e o inverno, cultivadas em Porto Amazona - PR, região de baixa ocorrência de frio.

\section{MATERIALEMÉTODOS}

O trabalho foi realizado com ramos de macieira 'Imperial Gala' coletados no período de abril a agosto de 2000, em pomar com 5 anos de idade, conduzido em plantio adensado $(4,0 \times 1,35 \mathrm{~m})$, na Fazenda Agropecuária Boutin, em Porto Amazonas - PR (25,55 latitude Sul, 49, $90^{\circ}$ de longitude Oeste e $795 \mathrm{~m}$ de altitude).

A quantificação do frio ocorrido na região durante o período estudado foi determinada segundo o método do número de horas de frio (HF) abaixo de $7,2^{\circ} \mathrm{C}$ e pelo método da conversão de temperaturas para unidades de frio (UF), baseado no modelo Carolina do Norte, utilizadas por Petri et al. (1996) (Tabela 1).

Os ramos com comprimento superior a $30 \mathrm{~cm}$ e com inserção e disposição espacial oblíqua foram coletados em sete épocas distintas: 19-04, 10-05, 31-05, 21-06, 12-07, 02-08 e 23-08. Após cada coleta, os ramos receberam ou não tratamento com frio suplementar de 1.440 horas à temperatura de $4 \mathrm{a} 7^{\circ} \mathrm{C}$ em geladeira.

A avaliação da dormência foi realizada por meio do teste

\footnotetext{
${ }^{1}$ (Trabalho 139/2003). Recebido: 03/10/2003. Aceito para publicação: 05/03/2004. Parte da tese do primeiro autor apresentada à Universidade Federal do Paraná para obtenção do título de Doutor em Ciências.

${ }^{2}$ Engenheiro Agrônomo, Dr., Professor Adjunto II do Centro de Ciências Agrárias e Ambientais da Pontifícia Universidade Católica do Paraná. Rodovia BR 376, km 14, CEP 83010-500 - São José dos Pinhais - PR. Fone: (41) 382-1454. ruy @ rla01.pucpr.br.

${ }^{3}$ Eng. Agrônomo, Dr., Professor de Fruticultura do Departamento de Fitotecnia e Fitossanitarismo da Universidade Federal do Paraná. Rua dos Funcionários, 1540, CEP 80035-050. Curitiba-Paraná. Fone: (41) 350-5650. flazan@ufpr.br.
} 
TABELA 1 - Modelo da conversão de temperaturas em unidades de frio adotada para quantificação e qualificação do frio ocorrido na região de coleta dos ramos de macieira, em Porto Amazonas-Paraná

Faixa de temperatura $\left({ }^{\circ} \mathrm{C}\right)$

Unidades de frio
0
0,5
1,0
0,5
0
$-0,5$
$-1,0$
$-1,5$
$-2,0$

biológico de estacas de nós isolados, realizado em sala de crescimento, à temperatura de $25^{\circ} \mathrm{C}$ e fotoperíodo de 16 horas, no Laboratório de Micropropagação de Plantas do Departamento de Fitotecnia e Fitossanitarismo da Universidade Federal do Paraná. A porção mediana dos ramos foi dividida em estacas de $7 \mathrm{~cm}$ de comprimento, mantendose nas mesmas apenas a gema superior. Os testes foram realizados com quatro repetições de 10 estacas, totalizando 40 estacas por tratamento.

As estacas foram avaliadas individualmente a cada dois dias, até um período máximo de 40 dias, de acordo com os parâmetros de crescimento "Ponta Verde" (PV) (aparecimento de modificações na coloração da gema, ficando esta com o ápice esverdeado) e "Gema Aberta" (GAb) (aparecimento de folhas abertas).

Com base nestes parâmetros, foram calculadas as seguintes variáveis:

- Tempo médio para brotação (TMB): número médio de dias passados entre a instalação do experimento em cada data e a detecção do estádio PV.

- Velocidade de brotação (VB): ocorrência de brotação das gemas em função do tempo para brotação dada pela equação $\mathrm{VB}=\sum\left(\mathrm{n}_{\mathrm{i}} / \mathrm{t}_{\mathrm{i}}\right)$ (gemas/dia) em que $n_{i}=$ número de gemas que atingiram o estádio PV no tempo "i”, e $t_{i}=$ tempo após instalação do teste $(\mathrm{i}=1 \rightarrow 40)$

- Taxa final de brotação (TF): porcentagem de estacas com gemas que atingiram o estádio PV.

- Taxa de brotações vigorosas (TBV): porcentagem das estacas com gemas no estádio PV que evoluíram até o estádio GAb no período analisado $[\mathrm{TBV}=(\%$ de estacas com gemas no estádio Gab $) \times 100 / \mathrm{TF}]$

- Tempo médio para aparecimento de folhas abertas (TMFA): número médio de dias passados entre o estádio PV e GAb.

$\mathrm{O}$ delineamento experimental adotado foi o de parcelas subdivididas no tempo, com o fator principal arranjado em blocos casualizados, com quatro repetições. A parcela principal foi o tratamento ou não com frio suplementar, e a subparcela foram as sete datas de coleta, totalizando 14 tratamentos. A comparação entre médias de tratamentos foi feita pelo teste de Tukey, ao nível de significância de $5 \%$.

\section{RESULTADOSE DISCUSSÃO}

Por meio da análise do tempo médio para brotação (TMB) das gemas no teste de estacas de nós isolados, observou-se que a dormência das gemas sem frio suplementar evoluiu de forma significativa entre as datas de 19-04 e 10-05 e, em seguida, permaneceu constante até junho. Em julho, ocorreu novo aumento, caracterizando um pico na curva do TMB. Este valor máximo corresponde à época de endodormência mais intensa, $\mathrm{e}$ a queda do TMB a partir deste ponto caracteriza o final da endodormência (Figura 1), conforme propuseram Carvalho et al. (2000) e Herter et al. (2001).

A baixa ocorrência em frio natural na região contribuiu para o estabelecimento de uma dormência profunda até julho, quando apenas 95 horas de frio abaixo de $7,2^{\circ} \mathrm{C}$ ou até mesmo unidades de frio negativas haviam sido acumuladas (Tabela 2). Embora ainda não tenha sido definido o requerimento em frio para a macieira da cultivar estudada, segundo Petri et al. (1996), a macieira da cultivar 'Gala', que originou a cultivar
'Imperial Gala', requer 600 horas de frio abaixo de $7,2^{\circ} \mathrm{C}$ para a superação da dormência, quantidade de frio muito além da ocorrida no pomar de macieiras estudado.

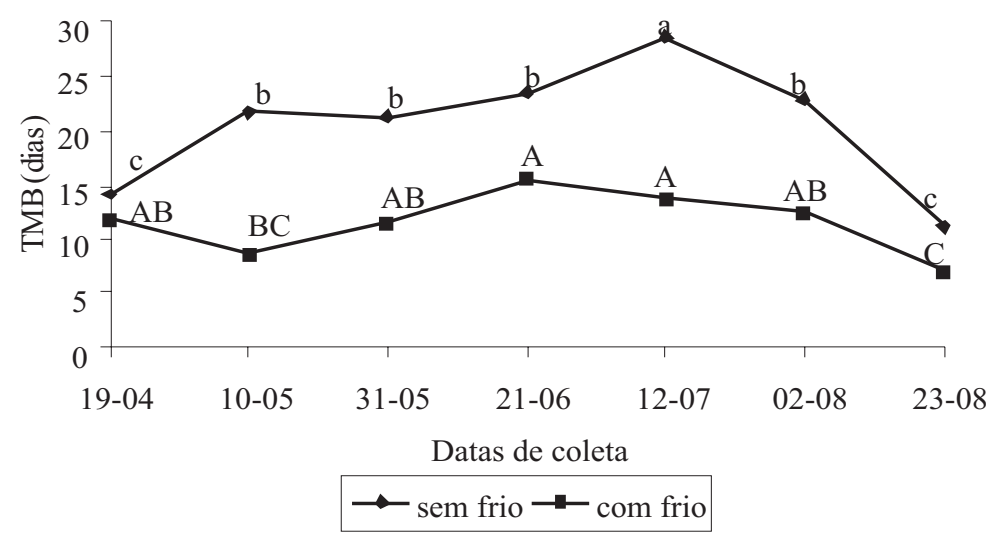

FIGURA 1 - Dinâmica do tempo médio para brotação (TMB) de gemas de um ano de idade de macieira 'Imperial Gala', cultivada em Porto Amazonas - Paraná, que receberam ou não 1.440 horas de frio suplementar de $4 \mathrm{a} 7^{\circ} \mathrm{C}$. Médias seguidas por letras distintas em cada curva diferem entre si, pelo teste de Tukey, ao nível de significância de 5\%.

TABELA 2 - Quantificação de frio ocorrido no período de maio a agosto de 2000 em Porto Amazonas - Paraná, segundo os modelos de horas de frio (HF) e unidades de frio (UF) baseada nos dados de temperatura horária, com ênfase nas datas de coleta dos ramos para avaliação.

\begin{tabular}{lcccc}
\hline Período/2000 & \multicolumn{2}{c}{ Horas de frio } & $7,2^{\circ} \mathrm{C}$ & \multicolumn{2}{c}{ Unidades de frio } \\
\cline { 2 - 5 } & No mês & Acumulado & No mês & Acumulado \\
\hline $1^{\circ}$ a 10-05 & 18 & 18 & $-63,5$ & $-63,5$ \\
10 a 31-05 & 39 & 57 & 98,0 & 34,5 \\
$31-05$ a 21-06 & 7 & 64 & $-74,5$ & $-40,0$ \\
$21-06$ a $12-07$ & 31 & 95 & $-46,0$ & $-86,0$ \\
$12-07$ a $02-08$ & 232 & 327 & 242,0 & 156,0 \\
$02-08$ a $23-08$ & 59 & 386 & 55,5 & 211,5 \\
\hline
\end{tabular}

O tratamento de 1.440 horas de frio suplementar de 4 a $7^{\circ} \mathrm{C}$ nas gemas alterou a dinâmica da curva preestabelecida, descaracterizando a ocorrência de um pico definido de TMB e mantendo os valores em níveis mais baixos que as gemas não tratadas. Esta resposta confirma a forte influência do tratamento com frio na evolução da dormência conforme afirmaram Flore \& Layne (1996), Pereira et al. (2001a) e Zanette et al. (2000). Esta influência foi particularmente importante no início de maio, quando as gemas não tratadas com frio entraram rapidamente em dormência, enquanto as que receberam frio, permaneceram aptas à brotação.

Mesmo com a continuidade da aplicação de frio suplementar, uma pequena elevação do TMB também ocorreu para as gemas coletadas até o mês de junho. Esta dinâmica pode estar relacionada com um efeito de outro fator ambiental importante nesta época, que é o fotoperíodo (Crabbé \& Barnola, 1996; Raven et al., 2001; Salisbury \& Ross, 1992). Adiminuição do fotoperíodo de abril a junho contribuiu para o estabelecimento de uma dormência que não pôde ser totalmente liberada unicamente pelo tratamento com frio suplementar. As folhas ainda presentes na planta, neste período, são os órgãos perceptores da diminuição do período de luz enviando sinais às gemas e ramos que determinam que uma estratégia de tolerância ao frio deve ser desenvolvida, baseada em alterações localizadas no metabolismo da planta.

Esta evolução da dormência ao longo do outono e inverno, atingindo valores máximos em julho, também alterou significativamente outras caraterísticas das gemas. No período de abril a julho, na ausência de frio, as gemas diminuíram a velocidade de brotação (VB) e a taxa final de brotação (TF) (Tabela 3), porém, mesmo as gemas que atingiram o estádio ponta verde em julho, não continuaram seu desenvolvimento (TBV $=0 \%$ ), demonstrando incapacidade de expandir folhas preexistentes nas gemas (Tabela 4). O efeito do corte da estaca acima da gema provocou 
uma resposta de início de brotação de algumas gemas a qual não se concretizou em formação de ramos bem desenvolvidos. Uma gema dormente é um ramo embrionário formado por um meristema apical, nós e entrenós não alongados e primórdios foliares (Raven et al., 2001; Salisbury \& Ross, 1992). O corte pode estimular o início de crescimento deste ramo embrionário, porém a expansão de folhas é um fenômeno dependente da ação de promotores do crescimento e do transporte de água a estes tecidos. Supõe-se que, nesta fase de dormência mais profunda, a poda interfere na capacidade de brotação da gema, mas não é um fator forte o suficiente para alterar totalmente o metabolismo na gema e nos tecidos adjacentes. Desta forma, não ocorre um suprimento de água e de reservas necessárias à manutenção do crescimento da gema estimulada.

TABELA 3 - Velocidade de brotação (VB) e taxa final de brotação (TF) resultantes do teste biológico com estacas de macieira 'Imperial Gala' com 1 ano de idade que receberam ou não 1.440 horas de frio suplementar de 4 a $7^{\circ} \mathrm{C}$, no período de abril a agosto de 2000, em Porto Amazonas - Paraná.

\begin{tabular}{lllllllll}
\hline \multirow{2}{*}{ Datas } & \multicolumn{3}{c}{ VB (gemas/dia) } & \multicolumn{4}{c}{ TF (\%) } \\
\cline { 2 - 9 } & \multicolumn{2}{c}{ Sem frio } & \multicolumn{2}{c}{ Com frio } & \multicolumn{2}{c}{ Sem frio } & \multicolumn{2}{c}{ Com frio } \\
\hline $19-04$ & $0,71 \mathrm{~A}^{*}$ & b & $0,92 \mathrm{BC}$ & a & $95,0 \mathrm{AB}$ & $\mathrm{a}$ & $95,0 \mathrm{~A}$ & $\mathrm{a}$ \\
$10-05$ & $0,11 \mathrm{BC}$ & b & $1,17 \mathrm{~B}$ & a & $20,0 \mathrm{C}$ & $\mathrm{b}$ & $97,5 \mathrm{~A}$ & $\mathrm{a}$ \\
$31-05$ & $0,28 \mathrm{~B}$ & $\mathrm{~b}$ & $0,85 \mathrm{C}$ & $\mathrm{a}$ & $55,0 \mathrm{~B}$ & $\mathrm{~b}$ & $100 \mathrm{~A}$ & $\mathrm{a}$ \\
$21-06$ & $0,08 \mathrm{BC}$ & $\mathrm{b}$ & $0,58 \mathrm{D}$ & $\mathrm{a}$ & $17,5 \mathrm{C}$ & $\mathrm{b}$ & $80,0 \mathrm{~A}$ & $\mathrm{a}$ \\
$12-07$ & $0,03 \mathrm{C}$ & $\mathrm{b}$ & $0,67 \mathrm{CD}$ & $\mathrm{a}$ & $7,5 \mathrm{C}$ & $\mathrm{b}$ & $87,5 \mathrm{~A}$ & $\mathrm{a}$ \\
$02-08$ & $0,08 \mathrm{BC}$ & $\mathrm{b}$ & $0,81 \mathrm{CD}$ & $\mathrm{a}$ & $17,5 \mathrm{C}$ & $\mathrm{b}$ & $97,5 \mathrm{~A}$ & $\mathrm{a}$ \\
$23-08$ & $0,92 \mathrm{~A}$ & $\mathrm{~b}$ & $1,58 \mathrm{~A}$ & $\mathrm{a}$ & $97,5 \mathrm{~A}$ & $\mathrm{a}$ & $100 \mathrm{~A}$ & $\mathrm{a}$ \\
\hline
\end{tabular}

*Médias seguidas por letras maiúsculas distintas nas colunas ou por letras minúsculas distintas nas linhas dentro de cada variável diferem entre si, pelo teste de Tukey, ao nível de significância de 5\%.

TABELA 4 - Tempo médio para aparecimento de folhas abertas (TMFA) e taxa de brotações vigorosas (TBV) de gemas de macieira 'Imperial Gala' que receberam ou não 1.440 horas de frio suplementar de $4 \mathrm{a} 7^{\circ} \mathrm{C}$, de abril a agosto de 2000, em Porto Amazonas - Paraná.

\begin{tabular}{|c|c|c|c|c|c|c|c|}
\hline \multirow[t]{2}{*}{ Datas } & \multicolumn{3}{|c|}{ TMFA (dias) } & \multicolumn{4}{|c|}{ TBV $(\%)$} \\
\hline & Sem fr & & Com frio & Sem fri & & Con & \\
\hline 19-04 & $5,0 \mathrm{AB}^{*}$ & $\mathrm{a}$ & $2,8 \mathrm{~A} \quad \mathrm{a}$ & $92,2 \mathrm{~A}$ & $\mathrm{a}$ & $84,5 \mathrm{~A}$ & $\mathrm{a}$ \\
\hline $10-05$ & $5,5 \mathrm{AB}$ & $\mathrm{a}$ & $4,7 \mathrm{~A}$ a & $75,0 \mathrm{AB}$ & $\mathrm{a}$ & $100 \mathrm{~A}$ & $\mathrm{a}$ \\
\hline $31-05$ & $2,9 \mathrm{~B}$ & $\mathrm{a}$ & $3,9 \mathrm{~A} \quad \mathrm{a}$ & $95,8 \mathrm{~A}$ & $\mathrm{a}$ & $100 \mathrm{~A}$ & $\mathrm{a}$ \\
\hline $21-06$ & $7,0 \mathrm{~A}$ & $\mathrm{a}$ & $4,2 \mathrm{~A} \quad \mathrm{~b}$ & $66,7 \mathrm{AB}$ & $\mathrm{a}$ & $92,0 \mathrm{~A}$ & $\mathrm{a}$ \\
\hline $12-07 * *$ & - & & 3,5 & $0,0 \quad \mathrm{C}$ & b & $90,8 \mathrm{~A}$ & $\mathrm{a}$ \\
\hline 02-08 & $4,8 \mathrm{AB}$ & $\mathrm{a}$ & $3,0 \mathrm{~A} \quad \mathrm{a}$ & $50,0 \mathrm{~B}$ & $\mathrm{~b}$ & $97,2 \mathrm{~A}$ & $\mathrm{a}$ \\
\hline $23-08$ & $2,5 \mathrm{~B}$ & $\mathrm{a}$ & $2,6 \mathrm{~A} a$ & $100 \mathrm{~A}$ & $\mathrm{a}$ & $97,5 \mathrm{~A}$ & $\mathrm{a}$ \\
\hline
\end{tabular}

*Médias seguidas por letras maiúsculas distintas nas colunas ou por letras minúsculas distintas nas linhas dentro de cada variável diferem entre si, pelo teste de Tukey, ao nível de significância de 5\%.

**Nesta data, nenhuma gema sem o tratamento com frio suplementar atingiu o estádio GAb. A análise estatística foi realizada sem esta data de coleta para a variável TMFA.

Baseado nestes dados, reforça-se a importância da utilização da variável Taxa de Brotações Vigorosas (TBV) nos testes de estacas de nós isolados como forma de avaliação da capacidade real de desenvolvimento da gema, diminuindo-se a interferência do corte da estaca como estimulador de início de desenvolvimento.

O tratamento com frio suplementar foi eficiente para aumentar a VB das gemas, a TF e a TBV, principalmente no período de dormência mais intensa, embora o TMB tenha sido um pouco elevado (Tabelas $3 \mathrm{e}$ 4). A indiferença entre as gemas tratadas e não tratadas com frio em relação à TF e à TBV no mês de abril é outro indicativo de que, neste período, a dormência foi fraca e o frio precoce não teve efeito na intensidade da dormência. Já no mês de maio, a redução da TF de gemas sem frio suplementar reforça a entrada na endodormência, embora a TBV tenha permanecido elevada.

\section{CONCLUSÕES}

1) A dormência mais intensa de gemas de um ano de macieira 'Imperial Gala' cultivada em região de baixa ocorrência de frio (Porto Amazonas, Paraná) ocorre em julho.

2) A aplicação de 1.440 horas de frio suplementar de 4 a $7^{\circ} \mathrm{C}$ altera a dinâmica da dormência das gemas, reduzindo o seu tempo médio de brotação.

3) A variável Taxa de Brotações Vigorosas (TBV) no teste de estaca de nós isolados é uma importante forma de avaliação da capacidade real de desenvolvimento da gema, diminuindo-se a interferência do corte da estaca como estimulador de início de desenvolvimento.

\section{REFERÊNCIAS BIBLIOGRÁFICAS}

BONHOMME, M.; RAGEAU, R.; GENDRAUD, M. ATP, ADP and NTP contents in vegetative and floral peach buds during winter: are they useful for characterizing the type of dormancy? In: VIÉMONT, J.D.; CRABBÉ, J. (Ed.) Dormancy in plants: from whole plant behaviour to cellular control. Cambridge: University Press, 2000. p.245-257.

CARVALHO, R. I. N.; ZANETTE, F.; DRON, C. Dormancy intensity in one, two and three year-old-buds of apple tree. Short Communications of Second International Symposium on Plant Dormancy, Angers, p.8-12, 2000.

CRABBÉ, J.; BARNOLA, P. A. New Conceptual Approach to Bud Dormancy in Woody Plants. In: LANG, G. A. (Ed.) Plant Dormancy: physiology, biochemistry and molecular biology. New York: CAB International, USA, 1996. p.83-113.

EREZ, A. Bud dormancy: a suggestion for the control mechanism and its evolution. In: VIÉMONT, J.-D.; CRABBÉ, J. (Ed.) Dormancy in plants: from whole plant behaviour to cellular control. Cambridge: University Press, 2000. p.23-33.

FLORE, J. A.; LAYNE, D. R. Prunus. In: ZAMSKI, E.; SCHAFFER, A. A. Photoassimilate distribution in plants and crops: source-sink relationships. New York: Marcel Dekker, 1996. p.825-849.

HERTER, F. G.; VERISSIMO, V.; GARDIN, J. P. P.; TREVISAN, R.; PEREIRA, I. S. Uso do "método biológico" na determinação da evolução da dormência em macieira e pereira. In: CONGRESSO BRASILEIRO DE FISIOLOGIA VEGETAL, 3., 2001, Ilhéus. Anais... CD-ROM.

LANG, G. A.; EARLY, J. D.; MARTIN, G. C.; DARNELL, R. L. Endo-, paraand ecodormancy: physiological terminology and classification for dormancy research. Hortscience, Alexandria, v.22, p.371-178, 1987.

LARCHER, W. Ecofisiologia vegetal. São Carlos: RiMa Artes e textos, 2000.531p.

MARQUAT, C.; VANDAMME, M.; GENDRAUD, M.; PÉTEL,G. Dormancy in vegetative buds of peach: relation between carbohydrate absorption potentials and carbohydrate concentration in the bud during dormancy and its release. Scientia Horticulturae, Amsterdam, v.79, p.151-162, 1999.

PEREIRA, J. E. S.; FORTES, G. R. L.; SILVA, J. B. Efeito da aplicação de baixa temperatura em plantas de macieira sobre o crescimento durante a aclimatização. Pesquisa Agropecuária Brasileira, Brasília, v.36, n.1, p.89-95, 2001a.

PEREIRA, J. E. S.; FORTES, G. R. L.; SILVA, J. B. Baixa temperatura para explantes do porta-enxerto de macieira 'Marubakaido' in vitro durante a aclimatização. Scientia Agrícola, Piracicaba, v.58, n.2, p.401405, 2001b.

PETRI, J. L.; PALLADINI, L. A.; SCHUCK, E.; DUCROQUET, J. P.; MATOS, C. S.; POLA, A. C. Dormência e indução da brotação de fruteiras de clima temperado. Florianópolis: EPAGRI, 1996. 110p. (Boletim Técnico, 75).

RAVEN, P. H.; EVERT, R. F.; EICHHORN, S. E. Biologia vegetal. Rio de 
Janeiro: Ed. Guanabara Koogan, 2001.906p.

SALISBURY, F. B.; ROSS C. W. Plant physiology. Califórnia: Wadsworth Publishing Company, 1992. 682p.

STAFSTROM, J. P. Regulation of growth and dormancy in pea axillary buds. In: VIÉMONT, J.-D.; CRABBÉ, J. (Ed.). Dormancy in plants: from whole plant behaviour to cellular control. Cambridge: University Press, 2000. p.331-346.

TAMURA, F.; TANABE, K.; ITAI, A.; TANAKA, H. Protein changes in the flower buds of japanese pear during breaking of dormancy by chiling or high-temperature treatment. Journal of the American Society for Horticultural Science, Alexandria, v.123, n.4, p.532-536, 1998.

ZANETTE, F.; CARVALHO, R. I. N.; DRON, C. Effect of low temperature on dormancy intensity in one, two and three year-old-buds of apple tree. Short Communications of Second International Symposium on Plant Dormancy, Angers, p.13-17, 2000. 